\title{
Comparison of auditory and visual conditioning stimuli in delay eyeblink conditioning in healthy young adults
}

\author{
Adam B. Steinmetz and Chad R. Edwards \\ Indiana University, Bloomington, Indiana \\ JoSEPH E. STEINMETZ \\ Ohio State University, Columbus, Ohio \\ AND \\ William P. Hetrick \\ Indiana University, Bloomington, Indiana
}

\begin{abstract}
Classical eyeblink conditioning (EBC) has been widely used to probe cerebellar function in humans and nonhuman mammals. Although the neural pathways governing behavior in this task are well understood and fairly discrete, it remains unclear in the human literature how conditioned stimuli (CSs) of different modalities (e.g., visual and auditory) influence the exhibition of conditioned responses (CRs). In the present study, therefore, CRs to a visual CS and an auditory CS were examined with the single-cue delay EBC procedure. An initial experiment $(N=61)$ was conducted to identify visual and auditory stimuli that had equal perceived intensities. Using these perceptually equivalent stimuli, a second group of 25 subjects completed auditory and visual EBC procedures in two testing sessions 5-8 days apart. Whereas the acquisition of CRs was similar between the CS modality conditions, the timing of the CRs differed such that earlier CR onset and peak latencies were associated with the visual CS. In addition, CR timing improved across testing sessions, as indicated by the later CR peak latencies exhibited during the second testing session, as compared with the first.
\end{abstract}

Classical eyeblink conditioning (EBC) has been widely used to probe cerebellar function in humans and nonhuman mammals. In the most common form of the EBC procedure, termed delay EBC, a conditioned stimulus (CS; e.g., a 400-msec tone) is paired with a coterminating unconditioned stimulus (US; e.g., a 50-msec corneal air puff). Repeated CS-US parings elicit the development of an adaptive conditioned blink response (CR), which occurs in healthy individuals just prior to the US. The shortdelay $\mathrm{EBC}$ procedure appears to be the purest assay of the functional integrity of the cerebellum, with the circuitry and synaptic mechanisms being well studied and identified in nonhumans (Christian \& Thompson, 2003; Kim \& Thompson, 1997; Steinmetz, 2000) as compared with other forms of EBC, such as trace conditioning (Christian \& Thompson, 2003; Woodruff-Pak \& Disterhoft, 2008). In addition, the cerebellar networks mediating this form of associative learning appear to be conserved across species (rats, Rogers, Britton, \& Steinmetz, 2001; humans, Gerwig, Kolb, \& Timmann, 2007). In addition, the short-delay procedure has been used to examine developmental and clinical conditions associated with cerebellar abnormalities and motor disruptions, such as aging (e.g., Woodruff-
Pak, Jaegre, Gorman, \& Wesnes, 1999; Woodruff-Pak \& Thompson, 1988), autism (Sears, Finn, \& Steinmetz, 1994), schizophrenia (e.g., Brown et al., 2005), and drug abuse (Skosnik et al., 2007). Although the neural pathways governing behavior in this task are well understood and fairly discrete (Kim \& Thompson, 1997; Steinmetz, 2000), it remains unclear in the human literature how CSs from different modalities (e.g., visual and auditory) influence the manifestation of CRs.

In a study comparing auditory and visual CSs during a discrimination EBC task in young rats (Days 17-31), Paczkowski, Ivkovich, and Stanton (1999) found similar rates of conditioned responses in the two modalities. However, auditory CSs were associated with significantly earlier CR onset and peak latencies and smaller CR amplitudes. In a more recent study by Lindquist, Vogel, and Steinmetz (2009), EBC was examined in rats, using an auditory and visual CS at three different CS-US interstimulus intervals (ISIs; 280, 580, and $880 \mathrm{msec}$ ). Shorter ISIs produced greater conditioning rates, with no changes in CR timing between modalities. At variance with the animal studies, the only human study to examine the effects of CS modality reported that healthy subjects exhib-

W. P. Hetrick, whetrick@indiana.edu 
ited significantly more CRs to a visual CS than to auditory CSs (Spain, 1966); however, timing properties of the CRs, such as onset and peak latencies, were not evaluated.

Taken together, human and nonhuman research has yielded conflicting evidence. Whereas animal studies failed to demonstrate differences in percentages of CRs, the only known human study showed facilitated CR acquisition to a visual cue. Because CS intensity is known to affect CR acquisition and timing (Scavio \& Gormezano, 1974), a key methodological concern with these studies is the fact that the auditory and visual stimuli were not matched on perceptual intensity. Thus, reported modality effects in humans and the lack thereof in nonhuman mammals could have been due to different stimulus intensities between CS modalities. It is important to equate the CS intensities in order to examine whether CSs of the same perceived intensity produce similar acquisition and timing of the $\mathrm{CR}$.

To address this issue, the present study employed two experiments, one to identify visual and auditory stimuli that were matched on perceived intensity and another to determine whether these visual and auditory stimuli, when used as CSs in separate testing sessions about 1 week apart, would result in similar rates of conditioning. Three primary hypotheses were tested. (1) On the basis of the results of Spain (1966), it was hypothesized that the subjects would exhibit more CRs to the visual CS than to the auditory CS. (2) On the basis of a nonhuman study (Paczkowski et al., 1999), it was predicted that the auditory CS would be associated with shorter CR onsets and peak latencies, as compared with the visual CS. (3) Finally, since prior work has demonstrated similar CR performance (i.e., percentage and timings of the response) when EBC testing has been repeated 1 week later using the same CS (Durkin, Prescott, Furchtgott, Cantor, \& Powell, 1993; Finkbiner \& Woodruff-Pak, 1991), as well as cross-modal savings when the CS changes modality at the second session (Campolattaro \& Freeman, 2009; Schreurs \& Kehoe, 1987), it was predicted that acquisition and timing of CRs would be similar during the second session of conditioning.

\section{EXPERIMENT 1 \\ Equating the Subjective Perceptual Intensity of Visual and Auditory Stimuli}

\begin{abstract}
Method
Subjects. The subjects were recruited from introductory psychology courses at Indiana University, Bloomington, provided written consent, and received course credit for their participation. After consent was obtained, the participants completed an audiology test to ensure that their hearing was within normal range (above $20 \mathrm{~dB}$, at 500,1000 , and $2000 \mathrm{~Hz}$ ). Sixty-one participants completed the procedure (mean age $=19.78$ years, $S D=1.76 ;$ males $=32$, females $=$ 29). The subjects reported no history of neurological problems, such as strokes or seizures, no history of learning disabilities, and no illicit drug use and had both normal hearing and normal or correctedto-normal vision.
\end{abstract}

Procedure. The subjects completed a task in which they were instructed to judge the perceived intensity of a light with respect to that of a tone. The tone, presented through foam ear inserts, was $1000 \mathrm{~Hz}$ ( $80 \mathrm{~dB}$ SPL) and did not vary in intensity throughout the procedure. The visual stimulus was presented on a cathode ray tube (CRT) that was $120 \mathrm{~cm}$ away from the subject's forehead. The luminance of the visual stimulus was manipulated by adjusting the grayscale of the stimulus against a black background to make the visual stimulus appear brighter or darker, depending on the subject's response to a question about the perceived intensity of the visual stimulus with respect to the auditory stimulus. The visual stimulus was presented on a 16-in. CRT. The subject sat $120 \mathrm{~cm}$ away from the CRT, and the visual angle of the rectangular visual stimulus was $34^{\circ}$. Both the auditory and visual stimuli were $400 \mathrm{msec}$ in duration.

The procedure involved a training trial block followed by two blocks of testing trials. On each trial, the tone was followed $2 \mathrm{sec}$ later by a visual stimulus. Following each trial, a rating prompt appeared on the CRT for $2 \mathrm{sec}$, asking the subject which stimulus appeared more perceptually intense. Response options were that the tone was more intense, that the light was more intense, or that the two stimuli were perceived as having the same intensity. The intensity of the visual stimulus on the next trial would then be modified by increasing or decreasing the luminance via adjustments of the grayscale until the subject reported that the stimuli appeared to have the same intensity. After it was reported that the stimuli were the same, the trial would be repeated with the same-intensity light. If two consecutive same-intensity judgments were made, the procedure would continue into the second block, which would start from a random light intensity. If, on the second consecutive trial, the same intensity was not reported, the procedure would continue until two consecutive same-intensity stimuli were selected. The subjects were tested in a sound-attenuated booth, which was dimly lit by a 7-W incandescent night-light bulb.

\section{Results}

Three subjects were excluded from the analyses because their responses were identified as extreme outliers by the SPSS boxplot outlier identification method (i.e., data points that were six or more quartiles from the upper or lower ends of the interquartile range), yielding a final group of 58 participants (mean age: 19.52 years $\pm 1.69 ; 31$ males, 27 females). The average grayscale value reported as matching the perceived intensity of the tone was 41.14 $( \pm 4.37)$ in Block 1 and $39.14( \pm 3.85)$ in Block 2. The average grayscale luminance of these two blocks $(40.14 \pm$ 4.16) was used for the visual CS in Experiment 2.

\section{EXPERIMENT 2 Auditory and Visual Eyeblink Conditioning}

\section{Method}

Subjects. The subjects were 25 young adults (mean age $=$ 20.73 years \pm 1.13 ; males $=10$, females $=15$ ) who were recruited from the local university community via fliers and were paid for their participation. Prospective subjects were first screened over the phone to ensure that they were between the ages of 18 and 35 years and had no history of neurological problems, such as strokes or seizures; no history of learning disabilities; no illicit drug use; and normal or corrected-to-normal vision. Once they were in the lab and informed consent had been obtained, the subjects completed an audiology test to ensure that their hearing was within normal range (above $20 \mathrm{~dB}$, at 500, 1000, and $2000 \mathrm{~Hz}$ ).

EBC stimuli and procedure. The subjects completed a 158 -trial delay EBC procedure on two occasions. The subjects were randomly assigned to first complete a procedure consisting entirely of either a visual or an auditory CS. Of the 25 subjects who completed the first EBC session, 20 returned 1 week later ( \pm 4 days) to complete a second EBC session in which a CS from the other modality was presented throughout this second session (i.e., subjects who were trained with the auditory CS in Session 1 were trained with a visual CS when they returned approximately 1 week later, and vice versa). 
During each session, 8 US-alone trials were initially presented (intertrial interval $[\mathrm{ITI}]=15 \mathrm{sec}$ ). Without interruption, the acquisition phase immediately followed and consisted of 10 trial blocks (mean ITI $=15 \mathrm{sec}$, range $=10-20 \mathrm{sec}$ ), each containing 10 paired trials. As in Experiment 1, the auditory CS was a $1000-\mathrm{Hz}$ tone $(80 \mathrm{~dB}$ SPL), and the visual CS, determined in the first experiment, had a luminance of $1.10 \times 10^{2} \mu \mathrm{W} / \mathrm{cm}^{2}, 4 \mathrm{ft}$ from the CRT. Both CSs were $400 \mathrm{msec}$ in duration and coterminated with a $50-\mathrm{msec}$ air puff. Without indication to the subject or interruption in the ITI, the procedure concluded with an extinction phase consisting of 25 CS-alone and 25 US-alone trials randomly presented across blocks (mean ITI $=15 \mathrm{sec}$; range $=10-20 \mathrm{sec}$ ).

Eyeblinks were recorded using pairs of electromyographic (EMG) electrodes ( $8 \mathrm{~mm} \mathrm{Ag} / \mathrm{AgCl}$; Model TD-23; Med Associates, St Albans, VT). Bipolar recording electrodes, with conductive gel applied, were placed on the orbicularis palpebrarum muscle below each eye. One electrode was placed $1 \mathrm{~cm}$ below the eyelid under the pupil, and the other $1 \mathrm{~cm}$ below the outer canthus. A ground electrode was placed on the forehead. All electrode impedances were maintained below $10 \mathrm{k} \Omega$. The US consisted of a 10 -p.s.i. (50-msec duration) puff of medical-grade air presented to the left eye with copper tubing affixed to eyeglass rims with flexible copper wire and positioned $1 \mathrm{~cm}$ away from the inner canthus of the eye. Foam ear inserts were used for presentation of the CS tones (E-A-RLINK, Aearo Company Auditory Systems, Indianapolis, IN). EMG data were continuously recorded at $2.5 \mathrm{kHz}$ with a Sensorium EPA- 6 bioamplifier (high-pass filter $=1 \mathrm{~Hz}, 12 \mathrm{~dB} /$ octave; low-pass filter = $300 \mathrm{~Hz}$, eighth order elliptic; gain $=5,000$ ) and were acquired using Neuroscan software (Version 4.2; El Paso, TX).

EBC data processing. Individual trials were epoched from the continuous EMG data file beginning $500 \mathrm{msec}$ before presentation of the CS (using Neuroscan Edit software) and were filtered (10-Hz high-pass filter; $6 \mathrm{~dB} /$ octave) before being rectified and smoothed using a 41-point Gaussian weighted moving average. Data were then entered into the Data-Munch software program for further analysis (King \& Tracy, 1999). For each subject, responses were recorded as blinks if the amplitude exceeded five standard deviations above the baseline (baseline window for each trial $=125 \mathrm{msec}$ before CS presentation). CRs were recorded if the blink occurred between 100 and $350 \mathrm{msec}$ after CS onset (corresponding to a period beginning $250 \mathrm{msec}$ before US onset). The onset latency was calculated as the point in time at which the CR exceeded five standard deviations from the baseline. The peak latency is the time point for the maximal value for that CR. Trials on which spontaneous blinks occurred within a window from $75 \mathrm{msec}$ before CS presentation to $25 \mathrm{msec}$ following $\mathrm{CS}$ onset were labeled bad trials and excluded from further analysis.

Statistical analysis. The primary dependent measures for the eyeblink procedure were percentage of CRs, CR onset latency, CR peak latency, CR amplitude, and unconditioned response (UR) amplitude. For each of these variables, the majority of the analyses were conducted with a repeated measures ANOVA with 2 levels of a modality factor (auditory, visual) and 10 levels of a withinsubjects block factor. The modality factor was a between-subjects factor when within-session effects were examined, whereas it was a within-subjects factor for analyses across sessions. An analogous analytical model was used to examine extinction data, except that the block factor had only 5 levels. For the primary dependent variables, effect sizes will be reported by $\eta_{\mathrm{p}}^{2}$, where small effect sizes are less than .06 , moderate effect sizes range from .06 to .14 , and large effect sizes are greater than .14 (Cohen, 1973). All statistical tests used an alpha level of $p<.05$ to determine significance (two-tailed). If Mauchly's test of sphericity was significant, Greenhouse-Geisser was used to determine significance. All tests were performed using the software package SPSS 14.0.

\section{Results}

First EBC session. For percentage of CRs across the session, a significant main effect of block was revealed
$\left[F(9,15)=10.06, p<.001, \eta_{\mathrm{p}}^{2}=.30\right.$; see Figure 1, top panel]. Neither the main effect of modality $\left(\eta_{\mathrm{p}}^{2}=.08\right)$ nor the modality $\times$ block interaction $\left(\eta_{\mathrm{p}}^{2}=.04\right)$ was significant. For CR onset latency, there was a main effect of modality $\left[F(1,23)=8.69, p=.007, \eta_{\mathrm{p}}^{2}=.27\right]$, indicating that CRs to visual CSs were earlier (i.e., further from US onset; 171.16 msec post-CS onset \pm 10.043 ), as compared with responses to auditory CSs (213.89 $\mathrm{msec}$ \pm 10.45 ) (see Figure 2A). There also was a main effect of block on CR onset $\left[F(9,15)=2.21, p=.05, \eta_{\mathrm{p}}^{2}=\right.$ .09], indicating that CR onset latencies increased across blocks. Similarly, a main effect of modality on CR peak latency $\left[F(1,23)=10.79, p=.003, \eta_{\mathrm{p}}^{2}=.32\right]$ indicated that the visual CS produced earlier peaks $(238.82 \mathrm{msec}$ $\pm 8.66)$ than did the auditory CS $(279.85 \mathrm{msec} \pm 9.01)$. There also was a main effect of block on CR peak latency $\left[F(9,15)=5.98, p<.001, \eta_{\mathrm{p}}^{2}=.21\right]$ (see Figure 2C) $\mathrm{CR}$ rise time (i.e., $\mathrm{CR}$ peak minus $\mathrm{CR}$ onset latency) was associated with a main effect of block $[F(9,15)=3.17$, $\left.p=.024, \eta_{\mathrm{p}}^{2}=.66\right]$, but no main effect of modality $\left(\eta_{\mathrm{p}}^{2}=\right.$ $.00)$ or modality $\times$ block interaction $\left(\eta_{\mathrm{p}}^{2}=.30\right)$ (see Figure 2E). For UR amplitudes, there was a main effect of block $\left[F(9,15)=23.96, p<.001, \eta_{\mathrm{p}}^{2}=.51\right]$, indicating decreasing UR amplitude across blocks, but not a main effect of modality or an interaction. No differences were found with CR amplitudes.

In the extinction phase, CRs associated with the visual CS (37.98\% CRs \pm 4.34$)$ were extinguished better than those associated with the auditory CS (70.30\% CRs \pm 4.51$)$ as indicated by a main effect of modality $\left[F(1,23)=26.71, p<.001, \eta_{\mathrm{p}}^{2}=.54\right]$ and a block $\times$ modality interaction $\left[F(4,20)=4.65, p=.001, \eta_{\mathrm{p}}^{2}=.61\right]$ (see Figure 1).

Second EBC session. For the percentage of CRs across the second session, there was a significant main effect of block $\left[F(9,10)=2.69, p=.033, \eta_{\mathrm{p}}^{2}=.13\right]$ and a block $\times$ modality interaction $[F(9,10)=2.605, p=.037$, $\left.\eta_{\mathrm{p}}^{2}=.13\right]$ (see Figure 1, bottom panel). However, the main effect of modality $\left(\eta_{\mathrm{p}}^{2}=.07\right)$ was not significant. There was a main effect of modality on CR onset $[F(1,18)=$ $\left.7.17, p=.015, \eta_{\mathrm{p}}^{2}=.29\right]$, indicating that CR onset latencies differed across-modalities, with the visual modality attributed to earlier CRs than the auditory CS. However, there were no significant effects with $\mathrm{CR}$ peak latency, $\mathrm{CR}$ rise time, CR amplitude, UR amplitude, and the extinction phase.

Comparison of EBC performance between subjects who received the auditory $C S$ during the first versus the second $\mathbf{E B C}$ session. In order to examine the effects of session after changing CS modality, EBC variables obtained from subjects who received the auditory $\mathrm{CS}$ in the first session were compared with those obtained from subjects who received the auditory CS in the second session. When percentages of CRs were compared, a significant main effect of block $[F(9,12)=4.50, p=.002$, $\left.\eta_{\mathrm{p}}^{2}=.18\right]$ and a block $\times$ session interaction $[F(9,12)=$ 4.62, $p=.001, \eta_{\mathrm{p}}^{2}=.19$ ] were obtained. Although the main effect of session was not significant $[F(1,20)=$ $\left.0.27, p=.61, \eta_{\mathrm{p}}^{2}=.01\right]$, for the subjects who completed the auditory CS condition during the second conditioning 


\section{Session 1}

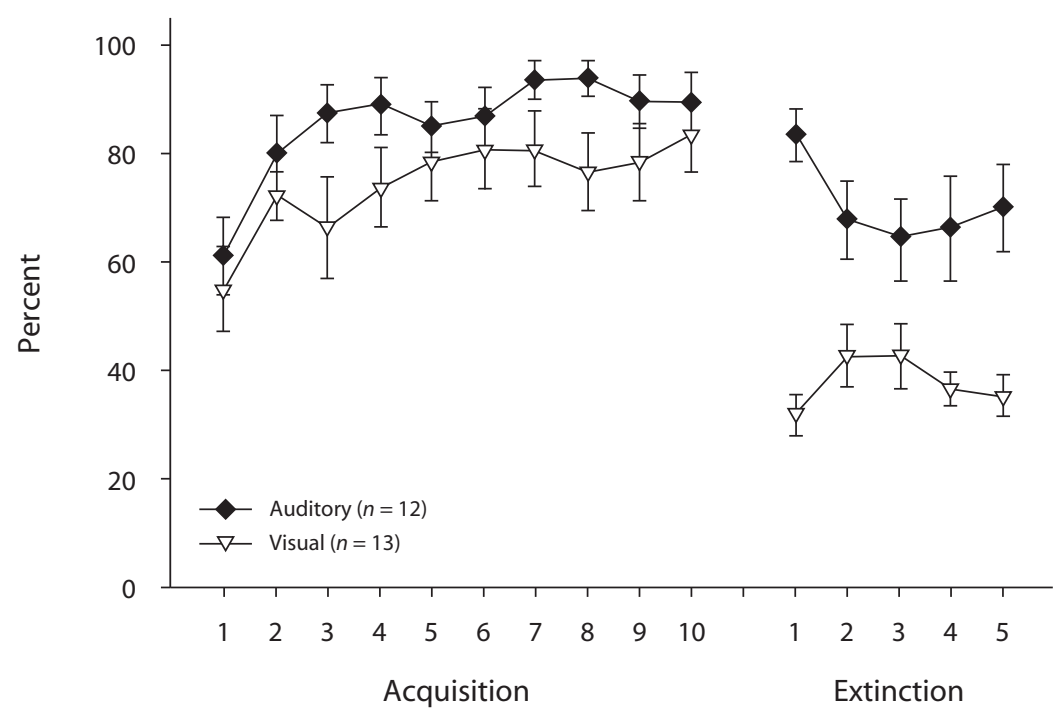

Session 2

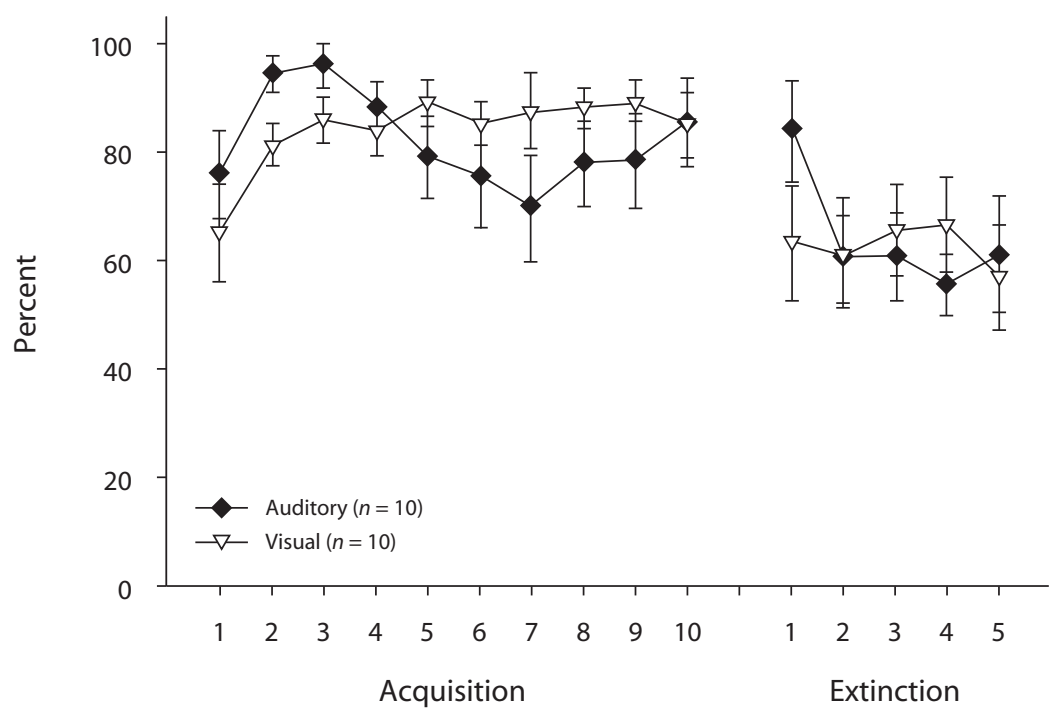

Figure 1. Percentage of conditioned responses for the auditory and visual conditioned stimuli (CSs) during Session 1 and Session 2. For Session 1, a main effect of block was observed $(p<.001)$, and for extinction, the two modalities were significantly different $(p<.001)$, with the visual CS extinguishing quicker. For Session 2 , a main effect of block was observed in both modalities $(p<.001)$. The auditory modality had a significant block $\times$ session interaction $(p=.032)$.

session, CRs began at $76 \%$, as compared with $61 \%$ for the group who received the auditory CS during the first conditioning session. The difference witnessed during the first block of conditioning also did not reveal a significant difference $\left[F(1,20)=1.89, p=.18, \eta_{\mathrm{p}}^{2}=.09\right]$. For those who received the auditory CS during the second session, a progressive increase in the percentage of CRs was seen across the first three trial blocks until a decrease in conditioning was observed (see Figure 1).
CR peak latencies occurred later during the second session (291.87 msec \pm 7.19 ), as compared with the first $(279.85 \mathrm{msec} \pm 6.56)$. A repeated measures ANOVA revealed a main effect of block $[F(9,12)=3.10, p=.025$, $\left.\eta_{\mathrm{p}}^{2}=.13\right]$ and a block $\times$ session interaction $[F(9,12)=$ 2.94, $\left.p=.032, \eta_{\mathrm{p}}^{2}=.13\right]$, although there was no main effect of session. No differences were revealed for CR onsets, CR amplitudes, CR rise time, or UR amplitudes (see Figure 2). 
Session 1

Session 2

A

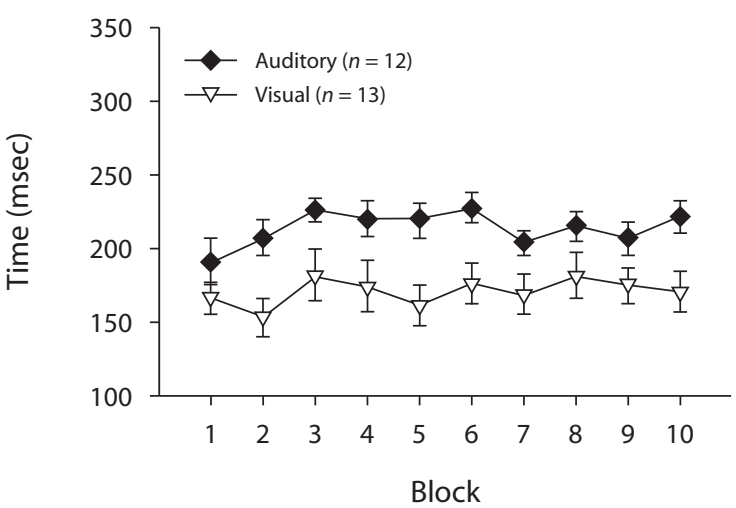

B

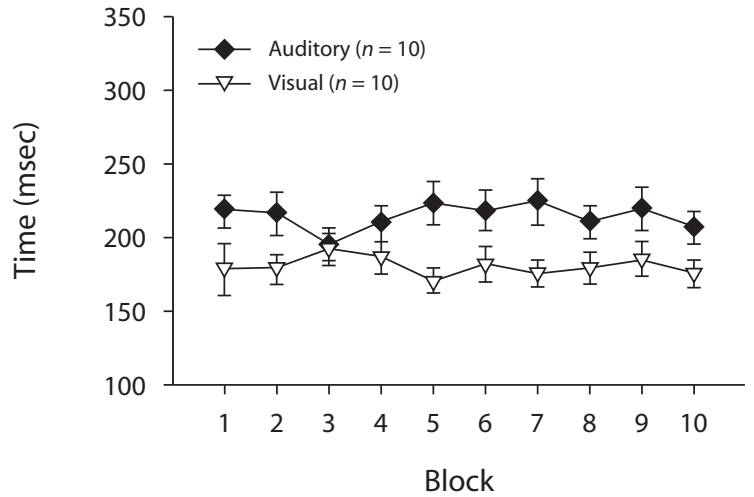

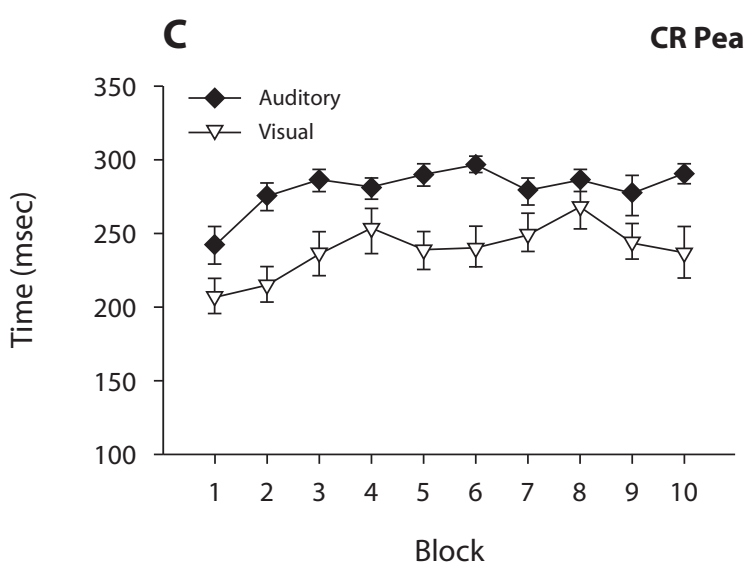
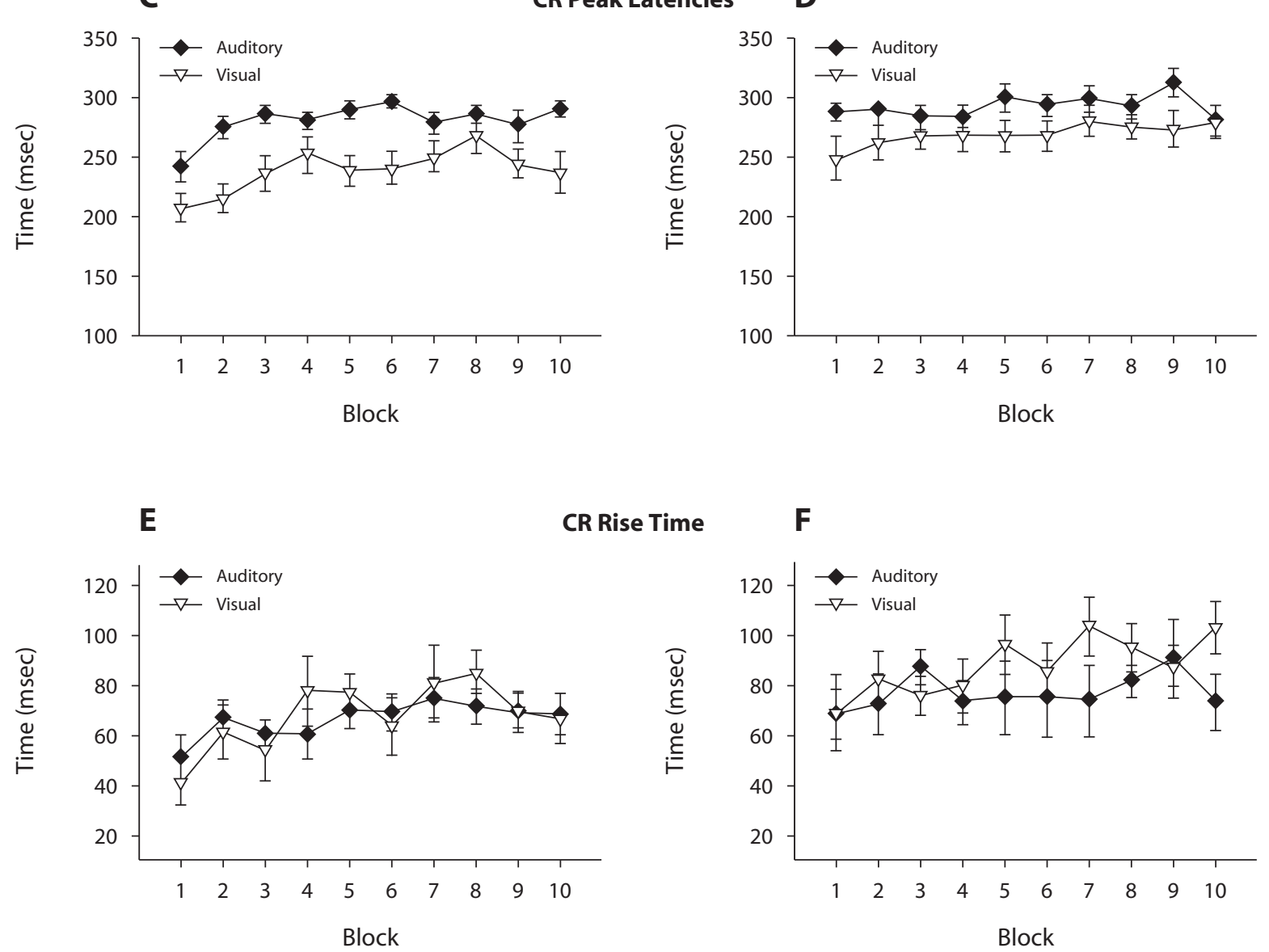

Figure 2. (A and B) Conditioned response (CR) onsets in the first (left panel) and second (right panel) sessions for the auditory and visual conditioned stimuli (CSs) with eyeblink conditioning. CR onsets are significantly later for the auditory CS $(p=$ $.007)$ than for the visual CS. (C and D) CR peak latencies are also significantly later for the auditory $(p<.001)$ than for the visual CS. ( $E$ and F) CR rise times (CR peak $-C R$ onset $\pm S E$ ) are shown for auditory versus visual CSs. However, there were no significant differences in CR rise times, which indicates that the modalities have different topographical characteristics. 


\section{Comparison of EBC performance between subjects who received the visual CS during the first versus the second EBC session. For percentage of CRs, there was a} main effect of block $\left[F(9,13)=5.83, p<.001, \eta_{\mathrm{p}}^{2}=.22\right]$, indicating increasing CRs across blocks. For the subjects who completed the visual CS during the second session, the CR peak latency was later $(268.90 \mathrm{msec} \pm 11.89)$, as compared with that observed for those who completed the visual CS during the first session (238.82 $\mathrm{msec} \pm 10.43$ ). Statistical analyses indicated a trend for the main effect of session $\left[F(1,21)=3.62 p=.07, \eta_{\mathrm{p}}^{2}=.15\right]$, and a main effect of block $\left[F(4.89,102.68)=3.92 p=.003, \eta_{\mathrm{p}}^{2}=\right.$ $.18]$, but no interaction. There were no differences in CR amplitudes, CR onsets, CR rise times, or UR amplitudes (see Figure 2).

First versus second session collapsed across modalities. To determine the effects of session order on eyeblink conditioning, independent of modality, the performance of the subjects was collapsed across modality within each of the two sessions. For percentage of CRs, a repeated measures ANOVA revealed a main effect of block $[F(9,35)=$ $\left.7.83, p<.001, \eta_{\mathrm{p}}^{2}=.15\right]$ and a block $\times$ session interaction $\left[F(9,35)=3.01, p=.010, \eta_{\mathrm{p}}^{2}=.065\right]$. This interaction indicated that learning differed across blocks within the two sessions, with percentage of CRs in the second session starting at a higher level and reaching an asymptote earlier than in the first session. However, there was no main effect of session $\left(\eta_{\mathrm{p}}^{2}=.008\right)$, indicating that learning occurred to a similar level overall in each session.

For CR peak latencies, there was a main effect of block $\left[F(9,35)=5.88, p<.001, \eta_{\mathrm{p}}^{2}=.12\right]$ and a main effect of session $\left[F(1,43)=4.30, p=.044, \eta_{\mathrm{p}}^{2}=.091\right]$. The block $\times$ session interaction did not reach significance $\left[F(9,35)=1.97, p=.073, \eta_{\mathrm{p}}^{2}=.044\right]$. Across blocks, the peak latencies moved closer to the US onset, and during the second session, these onsets were consistently closer to US onset, as compared with the first session. CR rise time revealed a main effect of block $[F(9,35)=2.69, p=$ $\left..020, \eta_{\mathrm{p}}^{2}=.402\right]$ and a main effect of session $[F(1,43)=$ $\left.6.07, p=.018, \eta_{\mathrm{p}}^{2}=.124\right]$. The CR rise time for the second session was smaller, indicating that actual duration of the blink was shorter. There were no differences regarding CR onset, CR amplitude, and UR amplitude.

\section{DISCUSSION}

This study was the first to examine the effects of CS modality on EBC, using stimuli matched on perceived perceptual intensity and a within-subjects design. The results indicated that when visual and auditory CS stimuli are matched on perceived intensity, comparable CR acquisition is achieved with auditory and visual CSs. However, a modality effect on CR timing was observed such that CR onset and peak latencies were earlier in the visual than in the auditory condition. In addition, performance on the second day of testing (about 1 week later) was characterized by improved CR peak latency adaptation (i.e., CRs appearing closer to US onset) during the second testing session about 1 week later, but similar CR acquisition, onset, and amplitude between CS modalities.

\section{CR Acquisition}

Consistent with findings from nonhuman studies (Lindquist et al., 2009; Paczkowski et al., 1999), the percentage of CRs associated with auditory and visual CSs did not statistically differ. However, this result is inconsistent with that in Spain (1966), which reported that healthy human subjects exhibited more CRs to a visual CS than to an auditory CS. Given that more intense CSs are associated with faster acquisition and better overall conditioning (Scavio \& Gormezano, 1974), perhaps the visual stimulus in the Spain study was perceptually stronger than the auditory stimulus. However, whether or not this was the case cannot be determined, because this issue was not addressed by Spain.

\section{CR Timing}

Contrary to what previous nonhuman animal studies have demonstrated (see Paczkowski et al., 1999), the present study showed that CR onset and peak latencies were earlier in relation to the US for the visual than for the auditory CS. The previously mentioned study showed that auditory CSs produced earlier CRs than did the visual CS. However, there is no evidence that CS intensities were matched in the previous studies, and the across-species differences in sensory processing cannot be ruled out as contributing to species differences. There is a possibility that the visual stimuli used in these studies were more intense and, thus, produced better timing. However, in the present study and the Paczkowski et al. study, no differences in the rise time were obtained. Rise time is described as the time between the onset and the peak of the response. The fact that the rise times were similar might indicate that CS modality affects the shape (topography) of the CR; this has been explained in past research as a topographical difference, or a difference in how the blink appears (Holland, 1977), that is modality dependent. CR topographic differences have previously been postulated to arise from interactions between learning and a large number of nonassociative factors, such as changes in auditory thresholds to the tone CS, magnitude of reflexive blinks to the air puff US, changes in modality of the CS, or changes in spontaneous blink rates (Martin \& Levey, 1969). The observed topographical differences suggest that while the speed of the eyelid closure was the same across CS modalities, the eyelid was held closed for a longer time before US onset in the auditory modality.

\section{Extinction}

Extinction of the visual CR occurred more rapidly than that of the auditory CR during the first session. One explanation for this could be that since the visual CS had less adaptive timing, the unlearning of a poorly learned response would occur more quickly. However, the percentages of CRs for the last 10 trials did not differ significantly between the two modalities, thus making this explanation less likely. Another possible explanation for this result could be that the visual CS allows extinction to occur at a faster rate. Extinction has been described as a process in which new inhibitory associations are formed while the original excitatory association is maintained. 
The mechanisms associated with extinction appear to be different to some extent from those associated with acquisition (Robleto, Poulos, \& Thompson, 2004; Robleto \& Thompson, 2008). These differences in mechanisms could be tapped by the different CS modalities, although extinction during the second session did not differ between the auditory and visual CSs.

\section{Multiple Testing}

The present study examined the relearning of the response over a 1-week period and across modalities. No group differences were observed in regard to overall percentages of CRs generated across Testing Sessions 1 and 2. This result is consistent with previous work showing no differences in the level of CRs in multiple days/ sessions of conditioning in humans (Woodruff-Pak \& Finkbiner, 1995) but conflicts with other research reporting further improvement in learning across multiple testing sessions in humans (Runquist \& Towart, 1965). The peak latency of the CR was closer to the UR in the second session than in the first session, contrary to past research (Woodruff-Pak \& Finkbiner, 1995). Early relearning during the second session was observed but was not dependent on the modality of the CS presented during the initial learning session or on the level of extinction in the first session. This indicates that after a week, timing of the CR was relearned rapidly. The fact that this recovery occurred after a modality change provides evidence of cross-modal savings. Another difference between the present study and previous studies (Runquist \& Towart, 1965; Woodruff-Pak \& Finkbiner, 1995) is that in the present study, extinction was included at the conclusion of the first EBC session. None of the previously mentioned human studies had an extinction period between testing sessions conducted between days. Previous studies in which cross-modal saving in animals has been examined, wherein an extinction phase was included, as occurred in the present study, have shown that these recoveries can occur, with reacquisition of the CR to the new CS being faster than that of initial learning (Macrae \& Kehoe, 1999). In the auditory training that occurred during the second session, the CR peak latencies still occurred later, as compared with CS onset. In contrast, $\mathrm{CR}$ peak latencies occurred earlier in the second session with the visual CS. These two pieces of information reveal that the visual CS produces early timing, as compared with the auditory CS, regardless of order of presentation.

\section{Limitations}

Several limitations should be mentioned. First, the subjects who completed the initial experiment to determine the perceptual equivalence of the CS stimuli were not the same as those who participated in the subsequent conditioning experiment. Thus, it is possible that the stimulus equivalence matching achieved with the first sample of subjects may not be generalized to the second sample. However, it would have impaired conditioning (i.e., through blocking) if the same subjects as those exposed to the testing stimuli in Experiment 1 subsequently had tried to learn the CS-US relationship. Second, when the subjects were asked to compare the intensities of the CSs in Experiment 1 , the respective sensory cortices in the cerebrum were involved in equating the intensities. However, actual associative learning occurs in the cerebellum. Thus, the intensity judgments made by the sensory cerebral cortex may not be the same as ones made by the cerebellum. However, since the purpose of this study was to compare the intensities of matched CSs in an associative learning procedure, this comparison was necessary. Another limitation is that to properly examine cross-modal savings in humans, two addition groups should be collected. These groups should not change modalities; thus, when they return after a week, they should complete the same modality as the one they received the first time. This would then allow a comparison of changes due to modality switch versus those due to there being a week between conditioning sessions.

\section{Conclusions}

Previous research in which an auditory EBC paradigm has been used has shown deficits in clinical groups, including schizophrenics (Brown et al., 2005) and drug abusers (Skosnik et al., 2007). However, few studies have used a visual CS in this paradigm to confirm and extend this research. One such study showed that schizophrenics could not condition to an auditory CS but could condition to a visual CS (Spain, 1966). A revisit and extension to this literature was needed in order to understand whether, given similar CS intensities, conditioning differences would be observed. The finding that the percentage of CRs did not differ between CS modalities suggests that for research environments where the presentation of auditory CSs may be problematic, such as during fMRI recordings, a visual CS may be used and will likely yield similar rates of eyeblink conditioning. In summary, when conditioning to visual and auditory CSs matched on perceived intensity is compared, the learning of the response is similar, but the CSs produce different timing (CR peak time and CR onset).

\section{AUTHOR NOTE}

This research was supported by a National Institute of Mental Health award to W.P.H. and J.E.S. (R01 MH074983-01). The authors thank Dale Sengelaub, Preston Garraghty, and Jim Craig. Correspondence concerning this article should be addressed to W. P. Hetrick, Department of Psychological and Brain Sciences, Indiana University, 1101 East 10th St., Bloomington, IN 47405 (e-mail: whetrick@indiana.edu).

\section{REFERENCES}

Brown, S. M., Keiffaber, P. D., Carroll, C. A., Vohs, J. L., Tracy, J. A., Shekhan, A., ET AL. (2005). Eyeblink conditioning deficits indicate timing and cerebellar abnormalities in schizophrenia. Brain \& Cognition, 58, 94-108. doi:10.1016/j.bandc.2004.09.011

Campolattaro, M. M., \& Freeman, J. H. (2009). Cerebellar inactivation impairs cross-modal savings of eyeblink conditioning. Behavioral Neuroscience, 123, 292-302. doi:10.1037/a0014483

Christian, K. M., \& Thompson, R. F. (2003). Neural substrates of eyeblink conditioning: Acquisition and retention. Learning \& Memory, 10, 427-455. doi:10.1101/1m.59603

CoHen, J. (1973). Eta-squared and partial eta-squared in communication science. Human Communication Research, 28, 473-490.

Durkin, M., Prescott, L., Furchtgott, E., Cantor, J., \& Powell, D. A. (1993). Concomitant eyeblink and heart rate classical conditioning in young, middle-aged, and elderly human participants. Psychology \& Aging, 8, 571-581. doi:10.1037/0882-7974.8.4.571 
FInkbiner, R. G., \& WoOdruff-PAK, D. S. (1991). Classical conditioning in adulthood: Effects of age and interstimulus interval on acquisition in the trace paradigm. Psychology \& Aging, 6, 109-111. doi:10.1037/0882-7974.6.1.109

Gerwig, M., Kolb, F. P., \& Timmann, D. (2007). The involvement of the human cerebellum in eyeblink conditioning. Cerebellum, 6, 38-57. doi: $10.1080 / 14734220701225904$

Holland, P. C. (1977). Conditioned stimulus as a determinant of the form of the Pavlovian conditioned response. Journal of Experimental Psychology, 3, 77-104. doi:10.1037/0097-7403.3.1.77

KIM, J. J., \& Thompson, R. F. (1997). Cerebellar circuits and synaptic mechanisms involved in classical eyeblink conditioning. Trends in Neurosciences, 20, 177-181. doi:10.1016/S0166-2236(96)10081-3

KIng, D. A. T., \& TrACY, J. (1999). DataMunch: A Matlab m-file collection for the analysis of trial-based spike and behavioral data. Available at www.novl.indiana.edu/Bdmunch/.

Lindquist, D. H., Vogel, R. W., \& Steinmetz, J. E. (2009). Associative and non-associative blinking in classically conditioned adult rats. Physiology \& Behavior, 96, 399-411. doi:10.1016/j .physbeh.2008.11.008

Macrae, M., \& KeHoe, E. J. (1999). Savings after extinction in conditioning of the rabbit's nictitating membrane response, Psychobiology, 27, 85-94

Martin, I., \& LeVey, A. B. (1969). The genesis of the classical conditioned response. Oxford: Pergamon.

Paczkowski, C., Ivkovich, D., \& Stanton, M. E. (1999). Ontogeny of eyeblink conditioning using a visual conditioned stimulus. Developmental Psychobiology, 35, 253-263. doi:10.1002/(SICI) 1098 -2302(199912)35:4

Robleto, K., Poulos, A. M., \& Thompson, R. F. (2004). Brain mechanisms of extinction of classically conditioned eyeblink response. Learning \& Memory, 11, 517-524. doi:10.1101/1m.80004

Robleto, K., \& Thompson, R. F. (2008). Extinction of a classically conditioned response: Red nucleus and interpositus. Journal of Neuroscience, 28, 2651-2658. doi:10.1523/JNEUROSCI.4781-07.2008

Rogers, R. F., Britton, G. B., \& Steinmetz, J. E. (2001). Learningrelated interpositus activity is conserved across species as studied during eyeblink conditioning in the rat. Brain Research, 905, 171-177. doi:10.1016/S0006-8993(01)02532-X
Runquist, W. N., \& Towart, E. (1965). Further evidence for intrasession decrements in eyelid conditioning. Psychological Reports, 17, 891-897.

Scavio, M. J., JR., \& GoRmeZANO, I. (1974). CS intensity effects on rabbit nictitating membrane conditioning, extinction and generalization. Pavlov Journal of Biological Science, 9, 25-34.

Schreurs, B. G., \& Kehoe, E. J. (1987). Cross-modal transfer as a function of initial training level in classical conditioning with the rabbit. Animal Learning \& Behavior, 15, 47-54.

Sears, L. L., FinN, P. R., \& S Teinmetz, J. E. (1994). Abnormal classical eye-blink conditioning in autism. Journal of Autism \& Developmental Disorders, 24, 737-751. doi:10.1007/BF02172283

Skosnik, P. D., Edwards, C. R., O'Donnell, B. F., StefFen, A., SteinMETZ, J. E., \& Hetrick, W. P. (2007). Cannabis use disrupts eyeblink conditioning: Evidence for cannabinoid modulation of cerebellardependent learning. Neuropsychopharmacology, 33, 1432-1440.

SpaIN, B. (1966). Eyelid conditioning and arousal in schizophrenic and normal participants. Journal of Abnormal Psychology, 71, 260-266. doi:10.1037/h0023596

Steinmetz, J. E. (2000). Brain substrates of classical eyeblink conditioning: A highly localized but also distributed system. Behavioural Brain Research, 110, 13-24. doi:10.1016/S0166-4328(99)00181-3

Woodruff-PaK, D. S., \& Disterhoft, J. F. (2008). Where is the trace in trace conditioning? Trends in Neurosciences, 31, 105-112. doi:10.1016/j.tins.2007.11.006

WoOdRUFF-PAK, D. S., \& Finkbiner, R. G. (1995). One-day retention of eyeblink classical conditioning and verbal free recall in young and older adults. Aging \& Cognition, 2, 108-127.

Woodruff-Pak, D. S., Jaeger, M. E., Gorman, C., \& Wesnes, K. A. (1999). Relationships among age, conditioned stimulus-unconditioned stimulus interval, and neuropsychological test performance. Neuropsychology, 13, 90-102. doi:10.1037/0894-4105.13.1.90

Woodruff-PaK, D. S., \& Thompson, R. F. (1988). Classical conditioning of the eyelid response in the delay paradigm in adults aged 18-83 years. Psychology \& Aging, 3, 219-229. doi:10.1037/0882 $-7974.3 .3 .219$

(Manuscript received March 4, 2009; revision accepted for publication June 15, 2009.) 